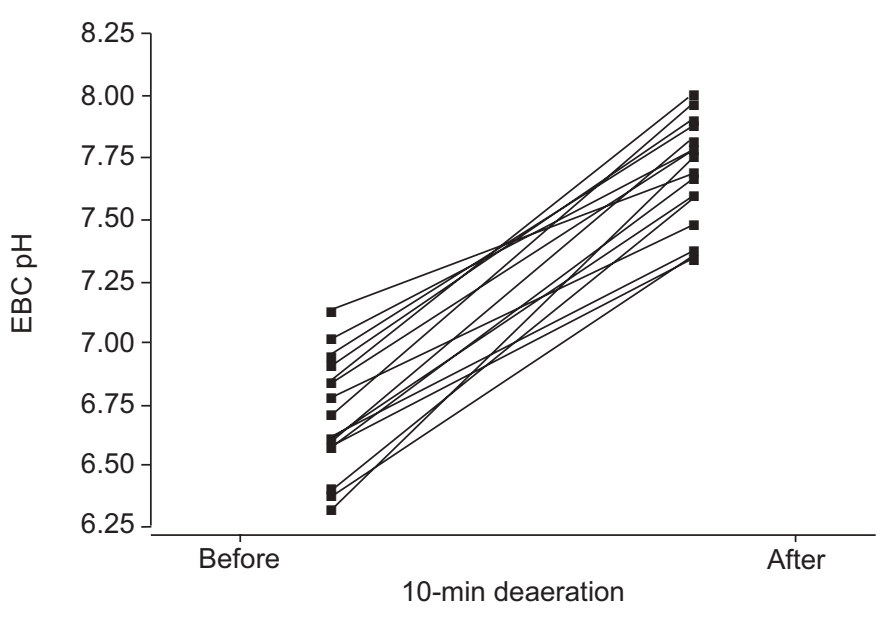

FIGURE 2. The effect of gas standardisation on exhaled breath condensate (EBC) $\mathrm{pH}$.

COPD patients. Our data are also in agreement with the suggestion by Z.L. Borrill and coworkers that a deaerationcaused $\mathrm{pH}$ change is a surrogate for $\mathrm{CO}_{2}$ concentration to some extent; however, a simple $\mathrm{pH}$ measurement cannot give an estimate of the remaining $\mathrm{CO}_{2}$ content that may still influence the $\mathrm{pH}$ reading.

In summary, argon deaeration decreases the concentration and the variability of exhaled breath condensate partial pressure of $\mathrm{CO}_{2}$, but there is always some remaining $\mathrm{CO}_{2}$ that may still be a confounding factor in $\mathrm{pH}$ assessment. $\mathrm{CO}_{2}$ content has a marked influence on exhaled breath condensate $\mathrm{pH}$ and, since exhaled breath condensate partial pressure of $\mathrm{CO}_{2}$ varies even after deaeration, it leaves some uncertainty in the exhaled breath condensate $\mathrm{pH}$ reading even after deaeration. It seems worthwhile to carry out some more research to define other potential modes of standardisation of this measurement, to learn more about the different factors that may influence exhaled breath condensate $\mathrm{pH}$ and the relationship between the $\mathrm{pH}$ of exhaled breath condensate and that of the airways.

\section{Horvath, B. Szili and T. Kullmann}

Dept of Pathophysiology, National Koranyi Institute for Pulmonology, Budapest, Hungary.

This study was supported by the Hungarian National Research Foundation (T43396).

\title{
A physiological-social score for triaging of pandemic influenza patients
}

\section{To the Editors:}

We read with interest the endorsement of "barefoot medicine" by EWIG et al. [1] in a recent issue of the European Respiratory Journal. As with NIEDERMAN et al. [2], we recognise the limitations of CURB-65 (confusion, urea $>7 \mathrm{mmol} \cdot \mathrm{L}^{-1}$, respiratory rate $\geqslant 30 \cdot \mathrm{min}^{-1}$, low blood pressure, and aged $\geqslant 65 \mathrm{yrs}$ ) scoring but the importance of its simplicity and ease of use. As part of the planning for a potential H5N1 influenza pandemic, using Dept of Health and Health Protection Agency projections [3], we have been forced to acknowledge that our urban emergency department, which normally sees $\sim 250$ patients $\cdot$ day $^{-1}$, will see 450 excess attenders $\cdot$ day $^{-1}$ with influenza symptoms at a pandemic peak. We aimed to develop a rapidly applicable, purely clinical scoring system for use in primary and secondary care, to identify those in need of hospital admission and to reassure those fit for discharge. We suggest that the ideal score should reflect acute physiological derangement, as well as accommodating age, comorbidities and social factors, and could be used to triage and track for admission, intensive care unit (ICU) treatment and mortality. We believe that our proposed system has gone some way towards addressing this.

We modified our hospital pandemic medical early warning score (PMEWS) [4] to include transcutaneous oxygen saturation. We also concur with EWIG et al. [1] and NIEDERMAN et al.
[2] that comorbidities and social factors have to be taken into account when making admission and discharge decisions, and our score incorporates an extra point for being aged $\geqslant 65$ yrs and another single point for any of the following: 1) social isolation (defined as living alone or having no fixed abode); 2) chronic disease (respiratory, cardiac, renal, diabetes mellitus or immunosuppression of any cause); or 3) performance status of limited activity or worse (modified Karnofsky $>2$ [5]).

The validation of 195 adult patients (101 aged $<65$ yrs) with a diagnosis of lower respiratory tract infection presenting to our emergency department (South Manchester University Hospitals Trust, Manchester, UK) between February and December 2005 showed good discrimination for the physiological section of the score, which was further improved by the addition of age and social factors. We retrospectively calculated PMEWS, CURB-65 and CRB-65 scores from emergency department medical and nursing notes, and constructed receiver-operating characteristics (ROC) curves for the prediction of admission (fig. 1). PMEWS without the transcutaneous oxygen saturation component is shown as we recognise that not all primary care providers will have access to a pulse oximeter.

We extended this to assess the value of the PMEWS score in predicting requirements for higher levels of care. Figure 2 shows the ROC curves for discrimination of need for high 


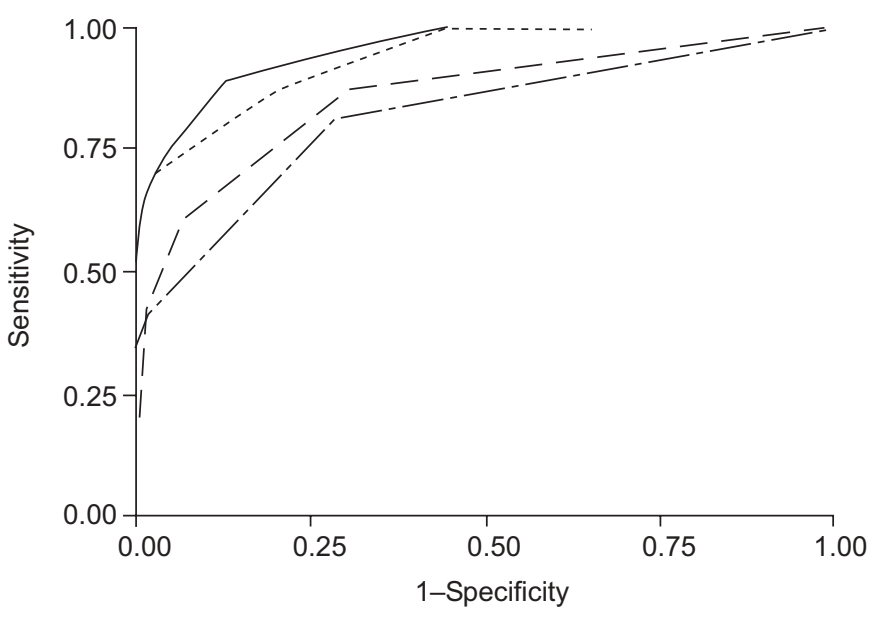

FIGURE 1. Receiver-operating characteristics (ROC) curve for admission to hospital. Diagonal segments are produced by ties. Data are presented as area under ROC curve ( $95 \%$ confidence interval for area under ROC curve). - pandemic medical early warning score (PMEWS) 0.953 (0.927-0.978); -----: PMEWS without transcutaneous oxygen saturation $0.937(0.906-0.967) ;---$ : CURB-65 (confusion, urea $>7 \mathrm{mmol} \cdot \mathrm{L}^{-1}$, respiratory rate $\geqslant 30 \cdot \mathrm{min}^{-1}$, low blood pressure, and aged $\geqslant 65 \mathrm{yrs}) 0.858$ (0.806-0.909); - - -: CRB-65 (confusion, respiratory rate $\geqslant 30 \cdot \mathrm{min}^{-1}$, low blood pressure, and aged $\left.\geqslant 65 \mathrm{yrs}\right) 0.818(0.760-0.877)$.

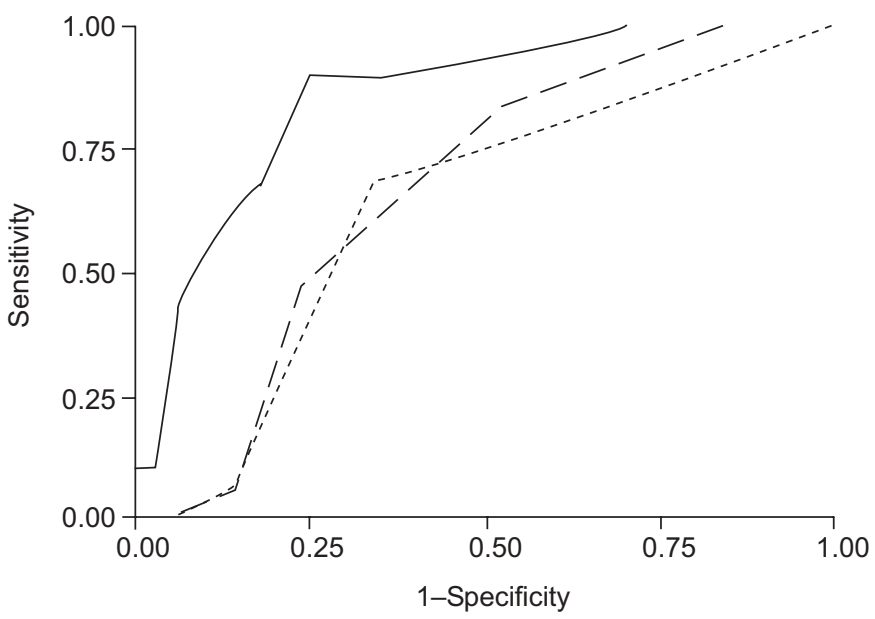

FIGURE 2. Receiver-operating characteristics (ROC) curve for admission to level 2/3 care. Diagonal segments are produced by ties. Data are presented as area under ROC curve ( $95 \%$ confidence interval for area under ROC curve). — pandemic medical early warning score $0.856(0.768-0.943)$; - - - : CURB-65 (confusion, urea $>7 \mathrm{mmol} \cdot \mathrm{L}^{-1}$, respiratory rate $\geqslant 30 \cdot \mathrm{min}^{-1}$, low blood pressure, and aged $\geqslant 65 \mathrm{yrs}$ ) 0.667 (0.549-0.785); -----: CRB-65 (confusion, respiratory rate $\geqslant 30 \cdot \mathrm{min}^{-1}$, low blood pressure, and aged $\left.\geqslant 65 \mathrm{yrs}\right) 0.631$ (0.497-0.765).

dependency or ICU care amongst 91 patients admitted with community-acquired pneumonia to our 855-bed secondary and tertiary care hospital from February to December 2005. A further number of patients $(n=19)$ were omitted from this analysis as invasive care and cardiopulmonary resuscitation were deemed inappropriate.

We suggest that as the pandemic medical early warning score is not disease specific, it can provide a more accurate assessment of need for hospital admission, clinical deterioration and improvement, and will be a valuable track and triage tool in the event of an influenza pandemic. Further validation is ongoing.

\section{K. Challen*, J. Bright ${ }^{\#}$, A. Bentley ${ }^{\#}$ and D. Walter*}

Depts of *Emergency Medicine, and "Respiratory Medicine, South Manchester University Hospitals Trust, Manchester, UK.

\section{REFERENCES}

1 Ewig S, Torres A, Woodhead M. Assessment of pneumonia severity: a European perspective. Eur Respir J 2006; 27: 6-8.

2 Niederman M, Feldman C, Richards G. Combining information from prognostic scoring tools for CAP: an American view on how to get the best of all worlds. Eur Respir J 2006; 27: 9-11.

3 UK Health Departments. UK Influenza Pandemic Contingency Plan. London, Department of Health, 2005.

4 Subbe C, Kruger M, Rutherford P, Gemmell L. Validation of a modified early warning score in medical admissions. Quarterly Journal of Medicine 2001; 94: 521-526.

5 British Thoracic Society Standards of Care Committee, Noninvasive ventilation in acute respiratory failure. Thorax 2002; 57: 192-211.

DOI: $10.1183 / 09031936.06 .00025106$

\section{From the authors:}

We welcome the contribution from K. Challen and co-workers concerning their pandemic medical early warning score (PMEWS) compared with CURB-65 (confusion, urea $>7$ $\mathrm{mmol} \cdot \mathrm{L}^{-1}$, respiratory rate $\geqslant 30 \cdot \mathrm{min}^{-1}$, low blood pressure, and aged $\geqslant 65 \mathrm{yrs}$ ) and CRB-65 (confusion, respiratory rate $\geqslant 30 \cdot \mathrm{min}^{-1}$, low blood pressure, and aged $\geqslant 65 \mathrm{yrs}$ ) as measures to determine hospital and intensive care unit admission for patients with lower respiratory tract infection (LRTI) and community-acquired pneumonia (CAP), but would urge caution before deciding which test to use in either CAP or pandemic influenza.

CURB-65 and CRB-65 have been developed for the assessment, at hospital admission, of patients with CAP and have now been validated in widely different populations [1]. Draft UK guidelines for the clinical management of pandemic influenza (www. dh.gov.uk/PublicationsAndStatistics/Publications/Publications PolicyAndGuidance/PublicationsPolicyAndGuidanceArticle/fs / en?CONTENT_ID $=4121753 \& c h k=Z$ XKxus) have used these scores as examples of how a severity scoring tool might be used in the light of this evidence base. However, neither the CURB scores nor PMEWS have actually been tested in patients with seasonal, let alone pandemic influenza where their operational characteristics may be different.

Other important issues are that CURB scores have been assessed in large patient populations compared with the 195 LRTI and 99 CAP patients described by K. Challen and co-workers. Important information is omitted by K. Challen and co-workers including: the definition of LRTI used; the proportion of LRTI patients admitted to hospital; and how many patients had missing variables. Without scrutiny of such information, we would not agree with these authors that they have shown that PMEWS can provide a more accurate assessment of need for hospital admission. 